\title{
Increased risk of abortion after frozen-thawed embryo transfer in women with polycystic ovary syndrome phenotypes A and D
}

\author{
Qiumin Wang ${ }^{1}$, Yanjun Zheng ${ }^{2}$, Ping Li $^{3}$, Guanqun Zhang ${ }^{4}$, Shanshan Gao ${ }^{4}$, Ze Wang ${ }^{4}$, \\ Baozhen $\mathrm{Hao}^{4}$, and Yuhua Shi ${ }^{4}$ \\ ${ }^{1}$ center for reproductive medicine, cheeloo college of medicine, Shandong University \\ ${ }^{2}$ Center for Reproductive Medicine, Cheeloo College of Medicine, Shandong University \\ ${ }^{3}$ Women and Children's Hospital, School of Medicine, Xiamen University, 10 Zhenhai \\ Road, 361003, Xiamen, China \\ ${ }^{4}$ Affiliation not available
}

September 25, 2021

\begin{abstract}
Abstract Objectives: To investigate pregnancy outcomes after frozen-thawed embryo transfer (FET) according to polycystic ovary syndrome (PCOS) phenotypes. Design: Retrospective study. Setting: University-based centre for reproductive medicine. Participants: 8903 patients who underwent FET between January 2017 and October 2019. Methods: All patients were divided into PCOS and control groups, with the former categorised into four phenotype groups (PCOS phenotypes A, B, C, D) based on Rotterdam criteria. All patient data were retrospectively collected and evaluated. Main outcome measures: Pregnancy outcomes after FET consisted of biochemical, clinical and ectopic pregnancies, abortion, premature delivery and live birth. Results: Women with PCOS phenotype A experienced an increased incidence of biochemical pregnancy, clinical pregnancy and premature delivery compared to those with PCOS phenotype $\mathrm{D}$ and in the control group $(\mathrm{P}<0.001, \mathrm{P}=0.005, \mathrm{P}=0.006$, respectively), while incidences of ectopic pregnancy and live birth were comparable between all groups $(\mathrm{P}>0.05)$. We found significantly higher abortion $(\mathrm{P}=0.010)$ and lower ongoing pregnancy $(\mathrm{P}=0.023)$ rates for women with $\mathrm{PCOS}$ phenotypes $\mathrm{A}$ and D compared to those in the control group. After adjusting for potential confounders, PCOS phenotypes A and D (vs. control) were associated with an elevated risk of abortion (adjusted odds ratio [OR], 1.476, 95\% confidence interval [CI], 1.077-2.024, P = 0.016; adjusted OR, 1.348, 95\% CI, 1.080-1.682, P = 0.008, respectively). Conclusions: For the first time, our study demonstrates that women with PCOS phenotypes A and D show an increased risk of abortion after FET.
\end{abstract}

\section{Introduction}

Polycystic ovary syndrome (PCOS) is considered a common endocrine disorder in women of reproductive age $^{1}$, affecting $6-21 \%$ of women worldwide ${ }^{2}$. Characteristics of PCOS include obesity, insulin resistance, hyperandrogenism, anovulation and polycystic ovaries ${ }^{3}$. Due to endocrine disorders and anovulation in women with PCOS, which lead to infertility ${ }^{4}$, such women usually require assisted reproductive technology (ART) to become pregnant.

Applying PCOS diagnostic criteria, four phenotypes are distinguished: phenotype A: coexistence of clinical hyperandrogenism/hyperandrogenemia (HA), oligomenorrhea/anovulation (OA) and polycystic ovaries (PCO); phenotype B: HA and OA without PCO; phenotype C: HA and PCO with regular ovulatory cycles; and phenotype D: OA coexisting with $\mathrm{PCO}^{2}$. Patients with different PCOS phenotypes show different ovarian responses to controlled ovarian hyperstimulation $(\mathrm{COH})^{5}$, which might contribute to different pregnancy outcomes $^{6}$. The previously published literature has mainly described pregnancy outcomes in patients with 
different PCOS phenotypes after fresh embryo transfer ${ }^{6,7}$; patients with PCOS are prone to ovarian hyperstimulation syndrome (OHSS) during or after $\mathrm{COH}^{8}$. Clinically, a fresh embryo transfer is usually cancelled to reduce the risk of $\mathrm{OHSS}^{9}$. However, it has been reported that frozen-thawed embryo transfer (FET) can not only reduce the risk of OHSS, but also improve ART outcomes ${ }^{10}$. Recently, a multi-centre randomised controlled trial of infertile women with PCOS suggested FET led to a higher rate of live births, and a lower risk of abortion than did fresh embryo transfer ${ }^{11}$. FET allows time for the use of preimplantation genetic technology ${ }^{12}$. Therefore, a considerable number of infertile patients choose to undergo FET. However, PCOS is associated with adverse pregnancy outcomes, including an increased risk of abortion, premature delivery, pre-eclampsia and even neonatal outcomes ${ }^{13,14}$. After removing the effect of $\mathrm{COH}$ on patients, studying the pregnancy outcomes of patients with different PCOS phenotypes after FET may better reflect the impact of different PCOS phenotypes on ART outcomes. Subsequent clinical management, which might optimise pregnancy outcomes, could then be carried out based on different PCOS phenotypes.

The aim of this study was to assess the effect of various PCOS phenotypes after FET on pregnancy outcomes.

\section{Materials and Methods}

\section{Study design and patients}

This study retrospectively collected and evaluated the clinical data of 8903 patients after FET at the Center for Reproductive Medicine, Cheeloo College of Medicine, Shandong University, who were patients between January 2017 and October 2019. All women were between the ages of 20 to 40 years with a body mass index (BMI) of no more than $35 \mathrm{~kg} / \mathrm{m}^{2}$, who underwent their first FET during their first in vitrofertilization/intracytoplasmic sperm injection cycle at our centre.

Women were excluded from this study if they (i) underwent frozen-thawed oocyte cycle or preimplantation genetic testing cycles, (ii) were diagnosed with premature ovarian insufficiency or a decreased ovarian reserve, (iii) had a history of unilateral oophorectomy, recurrent spontaneous abortion or severe intrauterine adhesion, (iv) had medical conditions that contraindicated assisted reproductive technology or pregnancy, and (v) were diagnosed with hypertension, diabetes, abnormal renal function, uterine malformation or abnormal parental karyotypes. This study was approved by the Ethics Committee of the Center for Reproductive Medicine, Cheeloo College of Medicine, Shandong University (2021-25).

All women were assigned to either PCOS or control groups. A diagnosis of PCOS was based on Rotterdam diagnostic criteria and if at least two of the following criteria were present: HA (hyperandrogenemia, defined as total testosterone levels above $48.1 \mathrm{ng} / \mathrm{dL}$, and hirsutism with a total score [?] 8 according to the FerrymanGallwey score); OA (defined as a delay of $>35$ days or $<$ eight spontaneous hemorrhagic episodes/years); PCO (defined as [?]12 small follicles measuring 2-9 mm in at least one ovary or ovarian volume [?]10 $\left.\mathrm{cm}^{3}\right)^{15}$. Patients with PCOS were categorised into four phenotype groups according to Rotterdam criteria as follows ${ }^{16}$ : phenotype A: $\mathrm{HA}+\mathrm{OA}+\mathrm{PCO}$; phenotype $\mathrm{B}$ : $\mathrm{HA}+\mathrm{OA}$; phenotype $\mathrm{C}$ : $\mathrm{HA}+\mathrm{PCO}$; and phenotype D: $\mathrm{OA}+\mathrm{PCO}$.

\section{Measurements}

Patient data were obtained from our centre records, included age, BMI, systolic blood pressure (SBP), diastolic blood pressure (DBP), duration and type of infertility, past medical history, a basic vaginal ultrasound, a basal hormone profile evaluation and outcomes of controlled ovarian hyperstimulation $(\mathrm{COH})$, as well as laboratory and clinical features of FET cycles. Blood samples of a basal hormone profile were collected for assessment on days $2-5$ of a spontaneous or progestin-induced menstrual cycle in all women. All the hormonal assays were performed in the laboratories of the Center for Reproductive Medicine, Cheeloo College of Medicine, Shandong University.

\section{Treatment}

All patients received a standard ovarian stimulation protocol (antagonist or long protocols), oocyte retrieval, fertilisation, embryo cultured and cryopreservedin vitro, and a luteal phase support protocol after embryo 
transfer (ET), according to a routine method. All patients who underwent FET in this study were found unsuitable for undergoing a fresh embryo transfer or did not get a viable neonate after undergoing a fresh embryo transfer. The outpatient physician chose the most appropriate protocol based on clinical indications of the prepared endometrium, which mainly included a natural cycle, hormonal replacement therapy cycle and ovulation induction cycle. Frozen blastocysts were thawed and transferred, and subsequently provided with luteal phase support according to different endometrial preparation programs.

\section{Outcomes of FET}

The primary outcomes after FET were pregnancy outcomes consisting of biochemical pregnancy, clinical pregnancy (CP), ectopic pregnancy, abortion, premature delivery and live birth (LB). A biochemical pregnancy was indicated by a serum human chorionic gonadotropin (HCG) level [?] 10 IU/L on day 14 after ET. A clinical pregnancy was considered detecting the presence of a gestational sac by ultrasonography on day 35 after ET. A diagnosis of ectopic pregnancy occurred when a developing blastocyst was found implanted outside the endometrial cavity. Abortion was defined as a clinical pregnancy lost before 28 weeks' gestation. Premature delivery was regarded as neonatal birth from the 28 th to 37 th week of gestation. Live birth was considered as the delivery of any viable neonate at 28 weeks of gestation or later.

\section{Statistical analysis}

Group means were compared using one-way analysis of variance (with the least significant difference posthoc test) for quantitative variables, and a chi-squared test for qualitative variables. Quantitative variables were expressed as the mean \pm standard deviation, and qualitative variables were presented as frequencies and percentages. Multivariate logistic regression analysis (backward: conditional) was performed to compare adjusted odds ratios (OR) and $95 \%$ confidence intervals (CI) for the effect of various PCOS phenotypes on pregnancy outcomes in FET, adjusted by the variates of $P<0.05$ in univariate logistic regression analysis.

All analyses were performed with the use of SPSS software (version 23.0). Two-sided $P$ values of less than 0.05 were considered to indicate statistically significant differences.

\section{Results}

\section{Patients' characteristics and outcomes of $\mathrm{COH}$}

A total of 8903 patients who underwent FET were enrolled in the present study, and categorised as 1887 PCOS women and 7016 non-PCOS women. Of the 1887 women with PCOS, 452 showed phenotype A $(\mathrm{HA}+\mathrm{OA}+\mathrm{PCO}, 23.95 \%$ of $\mathrm{PCOS}), 88$ presented with phenotype B (HA+OA, $4.66 \%$ of PCOS), 119 revealed phenotype $\mathrm{C}$ (HA+PCO, $6.31 \%$ of PCOS), and 1228 displayed phenotype $\mathrm{D}(\mathrm{OA}+\mathrm{PCO}, 65.08 \%$ of $\mathrm{PCOS})$.

Patient's baseline characteristics and ovarian responses of the four PCOS phenotypes and control group are listed in Table 1. A significant difference did not exist in histories of spontaneous abortion and premature delivery between the four PCOS phenotypes and control group $(P=0.922, P=0.495$, respectively). No significant differences in the type of infertility, fasting blood-glucose (FBG), gonadotropin dose and highquality embryos between the four PCOS phenotypes were found. Significant differences were observed with regard to age, BMI, SBP, DBP, duration of infertility, follicle-stimulating hormone, luteinizing hormone, total testosterone concentration (To), anti-Müllerian hormone (AMH), antral follicle count (AFC), the number of follicles of diameter [?] $14 \mathrm{~mm}$ and estradiol $\left(\mathrm{E}_{2}\right)$ levels on the HCG trigger day, and the number of retrieved oocytes between the four PCOS phenotype and control groups (all $P$-values were $<0.001$ ).

\section{FET cycle characteristics and pregnancy outcomes}

Table 2 summarises FET cycle characteristics and pregnancy outcomes for the four PCOS phenotype and control groups. A difference was found for the presence of a corpus luteum and endometrial thickness between the five groups $(P<0.001$ for both). Women with PCOS phenotype A showed an increased incidence of biochemical pregnancy, $\mathrm{CP}$ and premature delivery compared to those with PCOS phenotype $\mathrm{D}$ and in the control group $(P<0.001, P=0.005, P=0.006$, respectively), while the incidence of ectopic pregnancy and LB were comparable between the five groups $(P=0.596, P=0.397$, respectively). We also found a 
significantly higher abortion rate $(P=0.010)$ and lower ongoing pregnancy rate $(P=0.023)$ among PCOS phenotypes $\mathrm{A}$ and $\mathrm{D}$ compared to control groups.

After adjusting for potential confounders, PCOS phenotypes A and D (vs. control) were associated with an elevated risk of abortion (adjusted OR, 1.476, 95\% CI, 1.077-2.024, $P=0.016$; adjusted OR, 1.348, 95\% CI, 1.080-1.682, $P=0.008$, respectively). PCOS phenotype A (vs. control) was not a significant risk factor for a preterm delivery after adjusting results for potential confounders $(P=0.144$; Table 3 ). Potential confounders of abortion $(P<0.05$ in univariate logistic regression analysis) included age, BMI, duration and type of infertility, FBG, the number of retrieved oocytes and good quality embryos, and the presence or absence of a corpus luteum. Potential confounders of premature delivery $(P<0.05$ in univariate logistic regression analysis) included SBP, DBP, BMI, type of infertility, To, AMH, AFC and the number of transferred blastocysts in FET.

\section{Discussion}

\section{Main findings}

This study revealed a marked incidence of increased abortion in women of PCOS phenotype A and D groups after controlling for potential confounders. However, a finding of an elevated risk of premature delivery for the PCOS phenotype A group did not occur after controlling for potential confounders.

\section{Strengths and limitations}

In this study, we found, for the first time, that women with PCOS phenotypes A and D had an elevated risk of abortion after FET. This information will, for the first time, help clinicians develop individualised treatment plans to optimise ART outcomes. However, the investigation of several risk factors, such as environmental factors, physical activities and social factors, could not be included in this study. Due to the retrospective nature of this study, data on insulin resistance is lacking, and so was not included in the analysis. In addition, although we have the advantage of a large sample size, a large difference between groups was observed. Therefore, a further, large-scale and rigorous prospective validation study is necessary in future.

\section{Interpretation}

Abortion is a common complication of pregnancy. The aetiology of abortion is complex: obesity ${ }^{17}$, insulin resistance $^{18}$, hyperandrogenism ${ }^{19}$, the quality of oocytes and endometrial abnormalities might be associated with the occurrence of abortion ${ }^{20-22}$. As reported in prior studies, women with PCOS are associated with an increased risk of abortion ${ }^{23,24}$. In addition, Wang et al. ${ }^{25}$ found that the incidence of abortion was increased in women who underwent ART, with a possible mechanism related to corpus luteum insufficiency.

In our study, the incidence of abortion was significantly increased in PCOS phenotypes A and D, and with the coexistence of OA and PCOM in PCOS phenotypes A and D. We speculate that the combination of OA and PCOM might increase the risk of abortion by affecting oocyte quality. A study by Barnes and colleagues reported that oocyte maturation and the fertility rate of anovulatory women were significantly lower than those of regular cycling women; their embryo development ratio followed a similar trend ${ }^{26}$. Another study of anovulation in cows found that anovulation also leads to major shifts in gene expression in elongated conceptuses during preimplantation stages; transcripts involved with the control of energy metabolism and DNA repair were downregulated, whereas genes linked to apoptosis and autophagy were upregulated ${ }^{27}$. Furthermore, a recent study revealed decreased oocyte quality in PCOM due to the abnormal activation of one-carbon metabolism and hypermethylation of mitochondrial DNA ${ }^{28}$. These results support the above conjecture.

Moreover, we also observed that the rate for the presence of a corpus luteum (because of different endometrial preparation protocols for FET) in PCOS phenotypes A and D was significantly lower than that in controls, which might be another reason for the higher rate of abortion. In clinical practice, for PCOS women with OA, clinicians generally adopt a hormone replacement therapy (HRT) cycle to prepared the endometrium 
for $\mathrm{FET}^{29}$. Recently, Xu et al. ${ }^{30}$ noticed that HRT cycles were related to a higher abortion rate, which is consistent with the results of a prior study ${ }^{31}$. The corpus luteum is an important source of hormones in pregnant women ${ }^{12,32}$, but during endometrial preparation, a corpus luteum is absent in a HRT cycle. Additionally, administering exogenous hormone in a HRT cycle might increase the risk of thromboembolic events and could damage placentation, which may then lead to abortion ${ }^{33,34}$.

It is generally known that obesity has an undesirable impact on women's reproduction ${ }^{35}$. Obesity increases the rate of abortion ${ }^{36}$ and is an independent risk factor for abortion ${ }^{37,38}$. Obesity affects follicle development by affecting sex hormone secretion and metabolism ${ }^{35}$; other studies have found adverse effects of obesity on the quality of the embryo ${ }^{39}$ and endometrial receptivity ${ }^{40}$. In the present study, the BMI in PCOS phenotype A and D groups was significantly higher than that in the control group. Before the initiation of FET, obese women can reduce their weight to optimise pregnancy outcomes ${ }^{41}$. Interestingly, we also noticed that the age of the women in PCOS phenotypes A and D was lower than that of women in the control group. It is well documented that maternal age increases the incidence of abortion ${ }^{42,43}$. It is possible that other factors masked the effect of age on abortion. The average age of patients was $30.62+-4.07$ years in our study, which may have a relatively small impact on abortion. Previous studies found that an advanced age increased the risk of abortion, usually over the age of 35 or $38^{44,45}$. In addition, previous studies have suggested that $\mathrm{HA}$ is associated with increased rates of abortion ${ }^{46}$. However, this was not found in our study. A recent systematic review and meta-analysis also showed that HA does not increase the risk of abortion in patients with $\operatorname{PCOS}^{18}$.

Therefore, for women with PCOS phenotypes A and D, lifestyle interventions such as improved diet and increased exercise were used to reduce body weight before FET; natural or ovulation-induced cycles are recommended as a priority for endometrial preparation in FET, and an appropriately increased luteum phase supported. Additionally, pregnancy follow-up after obtaining a clinical pregnancy should be strengthened, and, with any sign of an abortion, treatment should be promptly provided.

\section{Conclusions}

In this study, we found, for the first time, that women with PCOS phenotypes A and D had an elevated risk of abortion after FET. We speculated that this was associated with the coexistence of OA and PCOM by affecting the quality of oocytes and embryos and the formation of a corpus luteum. The results of this study suggest that when performing FET, clinicians should individually manage women with PCOS phenotypes $\mathrm{A}$ and $\mathrm{D}$ to reduce the rate of abortion and achieve better pregnancy outcomes.

\section{Disclosure of interests}

The authors declare that they have no competing interests.

\section{Contribution to authorship}

YH Shi and QM Wang conceived and designed this study. QM Wang contributed to the statistical analyses, interpretation of data and drafting of the manuscript. YJ Zheng and P Li performed statistical analyses and participated in discussions. GQ Zhang acquired the data. SS Gao and Z Wang analysed and interpreted the data. YH Shi and QM Wang participated in the discussion and critically revised the manuscript. All authors read and approved the final manuscript.

\section{Details of ethics approval}

The institutional review board of the Center for Reproductive Medicine, Cheeloo College of Medicine, Shandong University approved this study (2021-25).

\section{Funding}

This study was supported by the National Key R\&D Program of China [2018YFC1003202 and 2016YFC1000604] and the Taishan scholar project special funds [No. ts201712103].

\section{Acknowledgments}


The authors thank clinicians, nurses, and laboratory staff for their contribution to this study. Moreover, the authors thank the infertile couples who participated in this study.

\section{Data availability}

The data and materials are available from the corresponding author on reasonable requests.

\section{REFERENCES}

1 Meier, R. K. Polycystic Ovary Syndrome. The Nursing clinics of North America 53 , 407-420, doi:10.1016/j.cnur.2018.04.008 (2018).

2 Lizneva, D., Suturina, L., Walker, W., Brakta, S., Gavrilova-Jordan, L. \& Azziz, R. Criteria, prevalence, and phenotypes of polycystic ovary syndrome. Fertility and Sterility 106 , 6-15, doi:10.1016/j.fertnstert.2016.05.003 (2016).

3 ACOG Practice Bulletin No. 194: Polycystic Ovary Syndrome.Obstetrics and gynecology 131, e157-e171, doi:10.1097/aog.0000000000002656 (2018).

4 Azziz, R., Carmina, E., Chen, Z., Dunaif, A., Laven, J. S., Legro, R. S. et al. Polycystic ovary syndrome. Nat Rev Dis Primers 2, 16057, doi:10.1038/nrdp.2016.57 (2016).

5 Cela, V., Obino, M. E. R., Alberga, Y., Pinelli, S., Sergiampietri, C., Casarosa, E. et al. Ovarian response to controlled ovarian stimulation in women with different polycystic ovary syndrome phenotypes. Gynecol Endocrinol 34 , 518-523, doi:10.1080/09513590.2017.1412429 (2018).

6 Ramezanali, F., Ashrafi, M., Hemat, M., Arabipoor, A., Jalali, S. \& Moini, A. Assisted reproductive outcomes in women with different polycystic ovary syndrome phenotypes: the predictive value of antiMullerian hormone. Reprod Biomed Online 32, 503-512, doi:10.1016/j.rbmo.2016.01.010 (2016).

7 Tal, R., Seifer, D. B., Khanimov, M., Malter, H. E., Grazi, R. V. \& Leader, B. Characterization of women with elevated antimullerian hormone levels (AMH): correlation of AMH with polycystic ovarian syndrome phenotypes and assisted reproductive technology outcomes. Am J Obstet Gynecol 211 , 59, e51-e58, doi:10.1016/j.ajog.2014.02.026 (2014).

8 Mourad, S., Brown, J. \& Farquhar, C. Interventions for the prevention of OHSS in ART cycles: an overview of Cochrane reviews. The Cochrane database of systematic reviews 1, Cd012103, doi:10.1002/14651858.CD012103.pub2 (2017).

9 Blumenfeld, Z. The Ovarian Hyperstimulation Syndrome. Vitam Horm 107 , 423-451, doi:10.1016/bs.vh.2018.01.018 (2018).

10 Roque, M., Haahr, T., Geber, S., Esteves, S. C. \& Humaidan, P. Fresh versus elective frozen embryo transfer in IVF/ICSI cycles: a systematic review and meta-analysis of reproductive outcomes. Hum Reprod Update 25 , 2-14, doi:10.1093/humupd/dmy033 (2019).

11 Chen, Z. J., Shi, Y., Sun, Y., Zhang, B., Liang, X., Cao, Y. et al. Fresh versus Frozen Embryos for Infertility in the Polycystic Ovary Syndrome. The New England journal of medicine 375 , 523-533, doi:10.1056/NEJMoa1513873 (2016).

12 Singh, B., Reschke, L., Segars, J. \& Baker, V. L. Frozen-thawed embryo transfer: the potential importance of the corpus luteum in preventing obstetrical complications. Fertil Steril 113 , 252-257, doi:10.1016/j.fertnstert.2019.12.007 (2020).

13 Roos, N., Kieler, H., Sahlin, L., Ekman-Ordeberg, G., Falconer, H. \& Stephansson, O. Risk of adverse pregnancy outcomes in women with polycystic ovary syndrome: population based cohort study. Bmj343 , d6309, doi:10.1136/bmj.d6309 (2011). 
14 Palomba, S., de Wilde, M. A., Falbo, A., Koster, M. P., La Sala, G. B. \& Fauser, B. C. Pregnancy complications in women with polycystic ovary syndrome. Hum Reprod Update 21, 575-592, doi:10.1093/humupd/dmv029 (2015).

15 Rotterdam ESHRE/ASRM-Sponsored PCOS consensus workshop group. Revised 2003 consensus on diagnostic criteria and long-term health risks related to polycystic ovary syndrome. Fertility and Sterility $\mathbf{8 1}$ , 19-25, doi:10.1016/j.fertnstert.2003.10.004 (2004).

16 Norman, R. J., Dewailly, D., Legro, R. S. \& Hickey, T. E. Polycystic ovary syndrome. The Lancet 370 , 685-697, doi:10.1016/s0140-6736(07)61345-2 (2007).

17 Qiu, M., Tao, Y., Kuang, Y. \& Wang, Y. Effect of body mass index on pregnancy outcomes with the freeze-all strategy in women with polycystic ovarian syndrome. Fertil Steril 112 , 1172-1179, doi:10.1016/j.fertnstert.2019.08.009 (2019).

18 Sun, Y. F., Zhang, J., Xu, Y. M., Cao, Z. Y., Wang, Y. Z., Hao, G. M.et al. High BMI and Insulin Resistance Are Risk Factors for Spontaneous Abortion in Patients With Polycystic Ovary Syndrome Undergoing Assisted Reproductive Treatment: A Systematic Review and Meta-Analysis. Frontiers in endocrinology 11 , 592495, doi:10.3389/fendo.2020.592495 (2020).

19 Zhang, Y., Zhao, W., Xu, H., Hu, M., Guo, X., Jia, W. et al.Hyperandrogenism and insulin resistanceinduced fetal loss: evidence for placental mitochondrial abnormalities and elevated reactive oxygen species production in pregnant rats that mimic the clinical features of polycystic ovary syndrome. The Journal of physiology597, 3927-3950, doi:10.1113/jp277879 (2019).

20 Song, J., Wang, X., Guo, Y., Yang, Y., Xu, K., Wang, T. et al.Novel high-coverage targeted metabolomics method (SWATHtoMRM) for exploring follicular fluid metabolome alterations in women with recurrent spontaneous abortion undergoing in vitro fertilization.Scientific reports $\mathbf{9}, 10873$, doi:10.1038/s41598-01947370-7 (2019).

21 Legro, R. S. Pregnancy considerations in women with polycystic ovary syndrome. Clin Obstet Gynecol 50 , 295-304, doi:10.1097/GRF.0b013e31803057ed (2007).

22 van der Spuy, Z. M. \& Dyer, S. J. The pathogenesis of infertility and early pregnancy loss in polycystic ovary syndrome. Best Pract Res Clin Obstet Gynaecol 18 , 755-771, doi:10.1016/j.bpobgyn.2004.06.001 (2004).

$23 \mathrm{Yu}, \mathrm{H}$. F., Chen, H. S., Rao, D. P. \& Gong, J. Association between polycystic ovary syndrome and the risk of pregnancy complications: A PRISMA-compliant systematic review and meta-analysis. Medicine $\mathbf{9 5}$, e4863, doi:10.1097/md.0000000000004863 (2016).

24 Zehravi, M., Maqbool, M. \& Ara, I. Polycystic ovary syndrome and reproductive health of women: a curious association. International journal of adolescent medicine and health, doi:10.1515/ijamh-2021-0031 (2021).

25 Wang, J. X., Norman, R. J. \& Wilcox, A. J. Incidence of spontaneous abortion among pregnancies produced by assisted reproductive technology.Hum Reprod 19, 272-277, doi:10.1093/humrep/deh078 (2004).

26 Barnes, F. L., Kausche, A., Tiglias, J., Wood, C., Wilton, L. \& Trounson, A. Production of embryos from in vitro-matured primary human oocytes. Fertil Steril 65 , 1151-1156, doi:10.1016/s0015-0282(16)58330-7 (1996).

27 Santos, J. E., Bisinotto, R. S. \& Ribeiro, E. S. Mechanisms underlying reduced fertility in anovular dairy cows.Theriogenology 86 , 254-262, doi:10.1016/j.theriogenology.2016.04.038 (2016).

28 Jia, L., Li, J., He, B., Jia, Y., Niu, Y., Wang, C. et al.Abnormally activated one-carbon metabolic pathway is associated with mtDNA hypermethylation and mitochondrial malfunction in the oocytes of polycystic gilt ovaries. Scientific reports 6 , 19436, doi:10.1038/srep19436 (2016). 
29 Aslih, N., Dorzia, D., Atzmon, Y., Estrada, D., Ellenbogen, A., Bilgory, A. et al. Ovulatory-Based FET Cycles May Achieve Higher Pregnancy Rates in the General Population and among Anovulatory Women.Journal of clinical medicine 10 , doi:10.3390/jcm10040703 (2021).

$30 \mathrm{Xu}$, H., Qiu, S., Chen, X., Zhu, S., Sun, Y. \& Zheng, B. D6 blastocyst transfer on day 6 in frozenthawed cycles should be avoided: a retrospective cohort study. BMC Pregnancy Childbirth 20 , 519, doi:10.1186/s12884-020-03224-z (2020).

31 Cerrillo, M., Herrero, L., Guillen, A., Mayoral, M. \& Garcia-Velasco, J. A. Impact of Endometrial Preparation Protocols for Frozen Embryo Transfer on Live Birth Rates. Rambam Maimonides medical journal 8, doi:10.5041/rmmj.10297 (2017).

$32 \mathrm{Liu}, \mathrm{X}$., Shi, W. \& Shi, J. Natural cycle frozen-thawed embryo transfer in young women with regular menstrual cycles increases the live-birth rates compared with hormone replacement treatment: a retrospective cohort study. Fertil Steril 113 , 811-817, doi:10.1016/j.fertnstert.2019.11.023 (2020).

33 Patel, S., Kilburn, B., Imudia, A., Armant, D. R. \& Skafar, D. F. Estradiol Elicits Proapoptotic and Antiproliferative Effects in Human Trophoblast Cells. Biol Reprod 93, 74, doi:10.1095/biolreprod.115.129114 (2015).

34 Hancke, K., More, S., Kreienberg, R. \& Weiss, J. M. Patients undergoing frozen-thawed embryo transfer have similar live birth rates in spontaneous and artificial cycles. J Assist Reprod Genet29 , 403-407, doi:10.1007/s10815-012-9724-z (2012).

35 Talmor, A. \& Dunphy, B. Female obesity and infertility. Best Pract Res Clin Obstet Gynaecol 29 , 498-506, doi:10.1016/j.bpobgyn.2014.10.014 (2015).

36 Metwally, M., Ong, K. J., Ledger, W. L. \& Li, T. C. Does high body mass index increase the risk of miscarriage after spontaneous and assisted conception? A meta-analysis of the evidence. Fertil Steril 90 , 714-726, doi:10.1016/j.fertnstert.2007.07.1290 (2008).

37 Bellver, J., Rossal, L. P., Bosch, E., Zuniga, A., Corona, J. T., Melendez, F. et al. Obesity and the risk of spontaneous abortion after oocyte donation. Fertil Steril 79, 1136-1140, doi:10.1016/s0015-0282(03)00176-6 (2003).

38 Zhou, H., Zhang, D., Luo, Z., Yang, A., Cui, N., Hao, G. et al. Association between Body Mass Index and Reproductive Outcome in Women with Polycystic Ovary Syndrome Receiving IVF/ICSI-ET. BioMed research international 2020 , 6434080, doi:10.1155/2020/6434080 (2020).

39 Metwally, M., Cutting, R., Tipton, A., Skull, J., Ledger, W. L. \& Li, T. C. Effect of increased body mass index on oocyte and embryo quality in IVF patients. Reprod Biomed Online 15 , 532-538, doi:10.1016/s14726483(10)60385-9 (2007).

40 Li, X., Ding, W., Liu, J. Y., Mao, Y. D., Huang, J., Wang, W.et al. [Effects of dyslipidemia on IVF/ICSI pregnancy outcome in patients with polycystic ovary syndrome]. Zhonghua Fu Chan Ke Za Zhi 53, 402-408, doi:10.3760/cma.j.issn.0529-567x.2018.06.008 (2018).

41 Lee, Y. S., Biddle, S., Chan, M. F., Cheng, A., Cheong, M., Chong, Y. S. et al. Health Promotion Board-Ministry of Health Clinical Practice Guidelines: Obesity. Singapore medical journal57 , 472, doi:10.11622/smedj.2016141 (2016).

42 Magnus, M. C., Wilcox, A. J., Morken, N. H., Weinberg, C. R. \& Haberg, S. E. Role of maternal age and pregnancy history in risk of miscarriage: prospective register based study. $B m j \mathbf{3 6 4}, 1869$, doi:10.1136/bmj.l869 (2019).

43 du Fosse, N. A., van der Hoorn, M. P., van Lith, J. M. M., le Cessie, S. \& Lashley, E. Advanced paternal age is associated with an increased risk of spontaneous miscarriage: a systematic review and meta-analysis. Hum Reprod Update 26 , 650-669, doi:10.1093/humupd/dmaa010 (2020). 
44 Li, J., Liu, X., Hu, L., Zhang, F., Wang, F., Kong, H. et al.A Slower Age-Related Decline in Treatment Outcomes After the First Ovarian Stimulation for in vitro Fertilization in Women With Polycystic Ovary Syndrome. Frontiers in endocrinology 10 , 834, doi:10.3389/fendo.2019.00834 (2019).

45 Weghofer, A., Munne, S., Chen, S., Barad, D. \& Gleicher, N. Lack of association between polycystic ovary syndrome and embryonic aneuploidy.Fertil Steril 88, 900-905, doi:10.1016/j.fertnstert.2006.12.018 (2007).

46 Yang, W., Yang, R., Yang, S., Li, J., Tu, B., Gao, C. et al.Infertile polycystic ovary syndrome patients undergoing in vitro fertilization with the gonadotropin-releasing hormone-antagonist protocol: role of hyperandrogenism. Gynecol Endocrinol34 , 715-718, doi:10.1080/09513590.2018.1431773 (2018).

Table 1. Baseline characteristics and ovarian responses of four PCOS phenotype and control groups

\begin{tabular}{llll}
\hline Variables & Phenotype A $(\mathrm{n}=452)$ & Phenotype B (n=88) & Phenotype C $(\mathrm{r}$ \\
\hline Age (years) & $28.89 \pm 3.43^{\mathrm{d}, \mathrm{e}}$ & $29.53 \pm 3.25^{\mathrm{e}}$ & $28.43 \pm 3.42^{\mathrm{d}, \mathrm{e}}$ \\
BMI $\left(\mathrm{kg} / \mathrm{m}^{2}\right)$ & $25.59 \pm 3.74^{\mathrm{b}, \mathrm{c}, \mathrm{d}, \mathrm{e}}$ & $24.66 \pm 3.54^{\mathrm{a}, \mathrm{e}}$ & $24.59 \pm 3.66^{\mathrm{a}, \mathrm{e}}$ \\
SBP & $117.27 \pm 11.88^{\mathrm{d}, \mathrm{e}}$ & $117.08 \pm 12.42^{\mathrm{e}}$ & $115.47 \pm 12.95^{\mathrm{e}}$ \\
DBP & $71.16 \pm 9.15^{\mathrm{c}, \mathrm{d}, \mathrm{e}}$ & $70.53 \pm 10.39^{\mathrm{c}, \mathrm{e}}$ & $67.53 \pm 9.47^{\mathrm{a}, \mathrm{b}, \mathrm{d}}$ \\
Duration of infertility & $4.09 \pm 2.33^{\mathrm{c}, \mathrm{e}}$ & $3.90 \pm 2.66$ & $3.55 \pm 2.86^{\mathrm{a}, \mathrm{d}}$ \\
Type of infertility, n (\%) & & & \\
Primary & $294 / 452(65.0)^{\mathrm{e}}$ & $54 / 88(61.4)^{\mathrm{e}}$ & $84 / 119(70.6)^{\mathrm{e}}$ \\
Secondary & $158 / 452(35)^{\mathrm{e}}$ & $34 / 88(38.6)^{\mathrm{e}}$ & $35 / 119(29.4)^{\mathrm{e}}$ \\
History of spontaneous abortion (\%) & $52 / 452(11.5)$ & $11 / 88(12.5)$ & $11 / 119(9.2)$ \\
History of premature delivery (\%) & $2 / 452(0.4)$ & $0 / 88(0.0)$ & $0 / 119(0.0)$ \\
FBG (mmol/L) & $5.26 \pm 0.44^{\mathrm{e}}$ & $5.34 \pm 0.45^{\mathrm{e}}$ & $5.30 \pm 0.45^{\mathrm{e}}$ \\
Basal FSH (IU/L) & $5.62 \pm 1.51^{\mathrm{b}, \mathrm{e}}$ & $6.01 \pm 1.45^{\mathrm{a}, \mathrm{d}}$ & $5.75 \pm 1.23^{\mathrm{e}}$ \\
Basal LH (IU/L) & $11.83 \pm 5.79^{\mathrm{b}, \mathrm{c}, \mathrm{d}, \mathrm{e}}$ & $8.13 \pm 4.52^{\mathrm{a}, \mathrm{e}}$ & $8.40 \pm 5.47^{\mathrm{a}, \mathrm{e}}$ \\
Basal To (ng/dL) & $62.50 \pm 14.35^{\mathrm{d}, \mathrm{e}}$ & $60.46 \pm 12.81^{\mathrm{d}, \mathrm{e}}$ & $61.66 \pm 12.03^{\mathrm{d}, \mathrm{e}}$ \\
AMH (ng/mL) & $11.50 \pm 4.18^{\mathrm{b}, \mathrm{c}, \mathrm{d}, \mathrm{e}}$ & $6.23 \pm 3.46^{\mathrm{a}, \mathrm{c}, \mathrm{d}, \mathrm{e}}$ & $8.78 \pm 4.29^{\mathrm{a}, \mathrm{b}, \mathrm{e}}$ \\
AFC & $34.51 \pm 12.03^{\mathrm{b}, \mathrm{c}, \mathrm{d}, \mathrm{e}}$ & $16.03 \pm 3.57^{\mathrm{a}, \mathrm{c}, \mathrm{d}}$ & $27.41 \pm 9.22^{\mathrm{a}, \mathrm{b}, \mathrm{d}}$ \\
Gn dose (IU) & $1791.77 \pm 968.01^{\mathrm{e}}$ & $1940.91 \pm 1009.77$ & $1759.87 \pm 814.84$ \\
No. of follicles of diameter [?]14 mm on HCG trigger day & $17.12 \pm 6.06^{\mathrm{b}, \mathrm{d}, \mathrm{e}}$ & $13.86 \pm 4.77^{\mathrm{a}, \mathrm{c}, \mathrm{d}, \mathrm{e}}$ & $16.73 \pm 4.72^{\mathrm{b}, \mathrm{e}}$ \\
E2 levels on HCG trigger day (pg/mL) & $6085.85 \pm 3355.60^{\mathrm{b}, \mathrm{d}, \mathrm{e}}$ & $5360.55 \pm 3117.31^{\mathrm{a}, \mathrm{e}}$ & $5879.19 \pm 3406.6$ \\
No. of retrieved oocytes & $19.04 \pm 8.77^{\mathrm{b}, \mathrm{d}, \mathrm{e}}$ & $15.23 \pm 7.29^{\mathrm{a}, \mathrm{c}, \mathrm{d}, \mathrm{e}}$ & $18.85 \pm 6.96^{\mathrm{b}, \mathrm{e}}$ \\
No. of good quality embryos & $5.36 \pm 3.46^{\mathrm{e}}$ & $5.19 \pm 3.61$ & $5.83 \pm 3.52^{\mathrm{e}}$ \\
\hline
\end{tabular}

AMH, anti-Müllerian hormone; AFC, antral follicle count; BMI, body mass index; DBP, diastolic blood pressure; FBG=fasting blood glucose; FSH=follicle-stimulating hormone; Gn, gonadotropin; HCG, human chorionic gonadotropin; IU, international units; LH, luteinizing hormone; PCOS, polycystic ovary syndrome phenotype; SBP, systolic pressure; To, total testosterone concentration

${ }^{a} P$-value $<0.05$ compared with phenotype $\mathrm{A}$

b $P$-value $<0.05$ compared with phenotype $\mathrm{B}$

c $P$-value $<0.05$ compared with phenotype $\mathrm{C}$

${ }^{\mathrm{d}} P$-value $<0.05$ compared with phenotype $\mathrm{D}$

e $P$-value $<0.05$ compared with control group

Table 2 FET cycle characteristics and pregnancy outcomes of four PCOS phenotype and control groups 


\begin{tabular}{lllll}
\hline Variables & Phenotype A $(\mathrm{n}=452)$ & Phenotype B $(\mathrm{n}=88)$ & Phenotype C $(\mathrm{n}=119)$ & Phenotype \\
\hline The presence of corpus luteum & & & & \\
Yes & $86 / 452(19.0)^{\mathrm{c}, \mathrm{e}}$ & $20 / 88(22.7)^{\mathrm{c}, \mathrm{e}}$ & $62 / 119(52.1)^{\mathrm{a}, \mathrm{b}, \mathrm{d}, \mathrm{e}}$ & $273 / 1228($ \\
No & $366 / 452(81.0)^{\mathrm{c}, \mathrm{e}}$ & $68 / 88(77.3)^{\mathrm{c}, \mathrm{e}}$ & $57 / 119(47.9)^{\mathrm{a}, \mathrm{b}, \mathrm{d}, \mathrm{e}}$ & $955 / 1228($ \\
No. of transferred blastocyst & $1.03 \pm 0.18$ & $1.07 \pm 0.25^{\mathrm{e}}$ & $1.04 \pm 0.20$ & $1.03 \pm 0.18$ \\
Endometrial thickness in FET (mm) & $9.1 \pm 1.4^{\mathrm{c}, \mathrm{e}}$ & $9.5 \pm 1.9$ & $9.6 \pm 1.5^{\mathrm{a}, \mathrm{d}}$ & $9.2 \pm 1.4^{\mathrm{c}, \mathrm{e}}$ \\
Biochemical pregnancy (\%) & $362 / 452(80.1)^{\mathrm{d}, \mathrm{e}}$ & $63 / 88(71.6)$ & $91 / 119(76.5)$ & $888 / 1228($ \\
CP (\%) & $321 / 452(71.0)^{\mathrm{d}, \mathrm{e}}$ & $53 / 88(60.2)$ & $82 / 119(68.9)$ & $797 / 1228($ \\
Ectopic pregnancy (\%) & $3 / 321(0.9)$ & $0 / 53(0.0)$ & $0 / 82(0.0)$ & $3 / 723(0.4)$ \\
Abortion (\%) & $66 / 321(20.6)^{\mathrm{e}}$ & $9 / 53(17.0)$ & $12 / 82(14.6)$ & $153 / 797(1)$ \\
Ongoing pregnancy (\%) & $252 / 321(78.5)^{\mathrm{e}}$ & $44 / 53(83)$ & $70 / 82(85.4)$ & $641 / 797(8$ \\
Premature delivery (\%) & $39 / 321(12.1)^{\mathrm{d}, \mathrm{e}}$ & $5 / 53(9.4)$ & $8 / 82(9.8)$ & $61 / 797(7.7$ \\
LB (\%) & $252 / 452(55.8)$ & $44 / 88(50.0)$ & $70 / 119(58.8)$ & $638 / 1228($ \\
\hline
\end{tabular}

The presence or absence of a corpus luteum was based on endometrial preparation protocols; that is, natural and ovulation induction cycles were considered to mean the formation of a corpus luteum, and a hormone replacement therapy cycle was regarded as meaning the absence of a corpus luteum.

$\mathrm{CP}$, clinical pregnancy; FET, frozen-thawed embryo transfer; LB, live birth

${ }^{\text {a }} P$-value $<0.05$ compared with phenotype A

b $P$-value $<0.05$ compared with phenotype $\mathrm{B}$

${ }^{c} P$-value $<0.05$ compared with phenotype $\mathrm{C}$

d $P$-value $<0.05$ compared with phenotype $\mathrm{D}$

e $P$-value $<0.05$ compared with control group

Table 3 Crude and adjusted ORs of various PCOS phenotypes for abortion and premature delivery

\begin{tabular}{lllll}
\hline & Crude OR (95\% CI) & $P$-values & Adjusted OR (95\% CI) & $P$-values \\
\hline Abortion (\%) & & & & \\
PCOS phenotype & & .010 & & .036 \\
PCOS-A & $1.447(1.091-1.920)$ & .010 & $1.476(1.077-2.024)$ & .016 \\
PCOS-B & $1.144(0.556-2.354)$ & .716 & $1.120(0.537-2.337)$ & .763 \\
PCOS-C & $0.958(0.517-1.778)$ & .893 & $1.209(0.642-2.275)$ & .557 \\
PCOS-D & $1.328(1.094-1.614)$ & .004 & $1.348(1.080-1.682)$ & .008 \\
Premature delivery (\%) & & & & \\
PCOS phenotype & & .006 & & .683 \\
PCOS-A & $1.932(1.355-2.756)$ & $<.001$ & $1.499(0.871-2.578)$ & .144 \\
PCOS-B & $1.455(0.575-3.683)$ & .429 & $1.178(0.440-3.152)$ & .745 \\
PCOS-C & $1.510(0.721-3.162)$ & .274 & $1.271(0.558-2.894)$ & .569 \\
PCOS-D & $1.158(0.869-1.542)$ & .316 & $1.053(0.737-1.504)$ & .776 \\
\hline
\end{tabular}

CI, confidence interval; OR, odds ratio; PCOS, polycystic ovary syndrome 